Hydroécol. Appl. (2001) Tome 13 Vol. 2, pp. 175-191

\title{
Étude des variations saisonnières du broutage zooplanctonique sur le peuplement phytoplanctonique de la retenue de barrage Al Massira (Maroc)
}

\section{Study of the seasonal variations of phytoplankton grazing by zooplankton species in the Al Massira reservoir (Morocco)}

\author{
Khadija Mokhliss, Mohammed Moncef \& Laïla Alaoui \\ Laboratoire d'Hydrobiologie - Faculté des Sciences - Université Chouaïb Doukkali BP 20 - \\ El Jadida - MAROC \\ e-mail :mdmoncef@yahoo.fr
}

Résumé. - La retenue Al Massira, qui fut mise en eau en 1979, constitue l'une des plus grandes retenues marocaines. Elle permet d'assurer une importante régularisation des débits de l'oued Oum Er-Rbia, naturellement irréguliers.

Des expériences, concernant l'évaluation de l'intensité de prédation zooplanctonique exercée sur les espèces phytoplanctoniques, ont été réalisées durant quatre périodes différentes, représentant les quatre saisons de l'année : octobre 1996, janvier, avril et juillet 1997.

A cet effet, nous avons utilisé la méthode de dénombrement des cellules algales ingérées par les espèces zooplanctoniques par unité de temps.

Les expériences réalisées ont consisté à incuber in situ deux enceintes à la profondeur $5 \mathrm{~m}$, pendant 8 heures, en absence (enceintes témoins) et en présence (enceinte expérimentale) de zooplancton.

Les résultats obtenus nous permettent de constater :

- Les bonnes performances de la technique utilisée dans la mesure où il y a une nette différence des densités algales dans les deux enceintes entre le début et la fin de l'expérience.

- Seules les espèces ayant à la fois une petite taille et un développement important sont préférentiellement sélectionnées par le peuplement zooplanctonique.

- La disponibilité des cellules algales consommables par les animaux planctoniques varie en fonction des saisons.

Mots clés. - Retenue, zooplancton, phytoplancton, broutage, variations saisonnières. 
Abstract. - The Al Massira reservoir constitutes on of the greatest reservoir in Morocco. It allows an important regulation of Oum Er Rbia River debit wich is naturally irregular. Experiments concerning the intensity of phytoplankton predation by zooplankton species, were realized during four different periods, representing the four saisons of the year : October 1996, January, April and July 1997.

The method used was based on the determination of the algal cells number grazed by zooplankton species per time unit.

Two enclosures were incubated in situ at $5 \mathrm{~m}$ depth for 8 hours : the first enclosure consisted of a mixture of several phytoplankton and zooplankton species, the second one was free of zooplankton.

The obtained results show that :

- The technique used presents good performance since a reel algal density difference is observed in two enclosures between the beginning and the end of the experiment.

- Only species with a small size and an important development are selected by zooplankton species.

- The availability of grazing algal cells by zooplankton is varying with saisons.

Keywords. - Reservoir, zooplankton, phytoplankton, grazing, seasonal variations.

\section{INTRODUCTION}

L'évaluation des flux de la matière au sein de la chaîne trophique, occupe actuellement une place dominante en écologie. Dans ce domaine, il existe en limnologie, un nombre important de travaux, surtout à l'interface phytoplancton-zooplancton : Vijverberg, 1976 ; Coveney et al., 1977 ; Kiorboe, 1985 ; Sager et al., 1990 ; Northcote et al., 1990 ; Paul et al., 1995.

L'étude du broutage (grazing en terme anglo-saxon) consiste à étudier la quantité de matière végétale ingérée par le zooplancton herbivore en un temps donné. La plupart des mesures qui ont été réalisées avaient en fait pour but d'étudier la nutrition des crustacés en relation avec la quantité et la qualité de nourriture disponible : Waleed et al., 1993 ; Mon- cef, 1993 ; Moncef et al., 1994 ; Tifnouti et al., 1994. D'autres chercheurs ont mesuré le broutage chez les rotifères (Oulad Ali, 1988).

Trois méthodes sont couramment utilisées pour quantifier le broutage du zooplancton sur le phytoplancton :

- La mesure de la fluorescence intestinale des animaux prélevés dans le milieu naturel (Mackas \& Bohrer, 1976) ;

- La mesure de la radioactivité du zooplancton après une brève incubation en présence d'algues marquées (Haney, 1971) ;

- Le dénombrement des algues dans des enceintes après incubation in situ, en absence et en présence de zooplancton (Porter, 1972).

Nous avons opté pour cette dernière méthode qui, malgré sa longue 
mise en œuvre, est performante dans la mesure où la différence entre les densités algales, entre les temps initial et final, est généralement significative dans les enceintes d'incubation (Moncef et al., 1994) et présente l'éventuelle sélectivité du broutage (Mourelatos, 1989).

Si les travaux sur le broutage du phytoplancton par le zooplancton sont relativement nombreux à travers le monde, au Maroc, les études dans ce domaine restent très rares (Moncef, 1993; Moncef et al., 1994 ; Fahde, 1994 ; Malki, 1994). Nous avons apporté une nouvelle contribution à ce sujet grâce à des expérien- ces de broutage aux quatre saisons de l'année (octobre 1996, janvier 1997, avril 1997 et juillet 1997).

\section{MATÉRIEL ET MÉTHODES}

\subsection{Site et station d'étude}

La retenue Al Massira, mise en eau en février 1979, est considérée comme la pièce maîtresse du plan d'aménagement du bassin versant de l'oued Oum Er Rbia. Elle se situe dans la province de Settat, à $120 \mathrm{Km}$ au sud-est de Casablanca (Fig. 1).

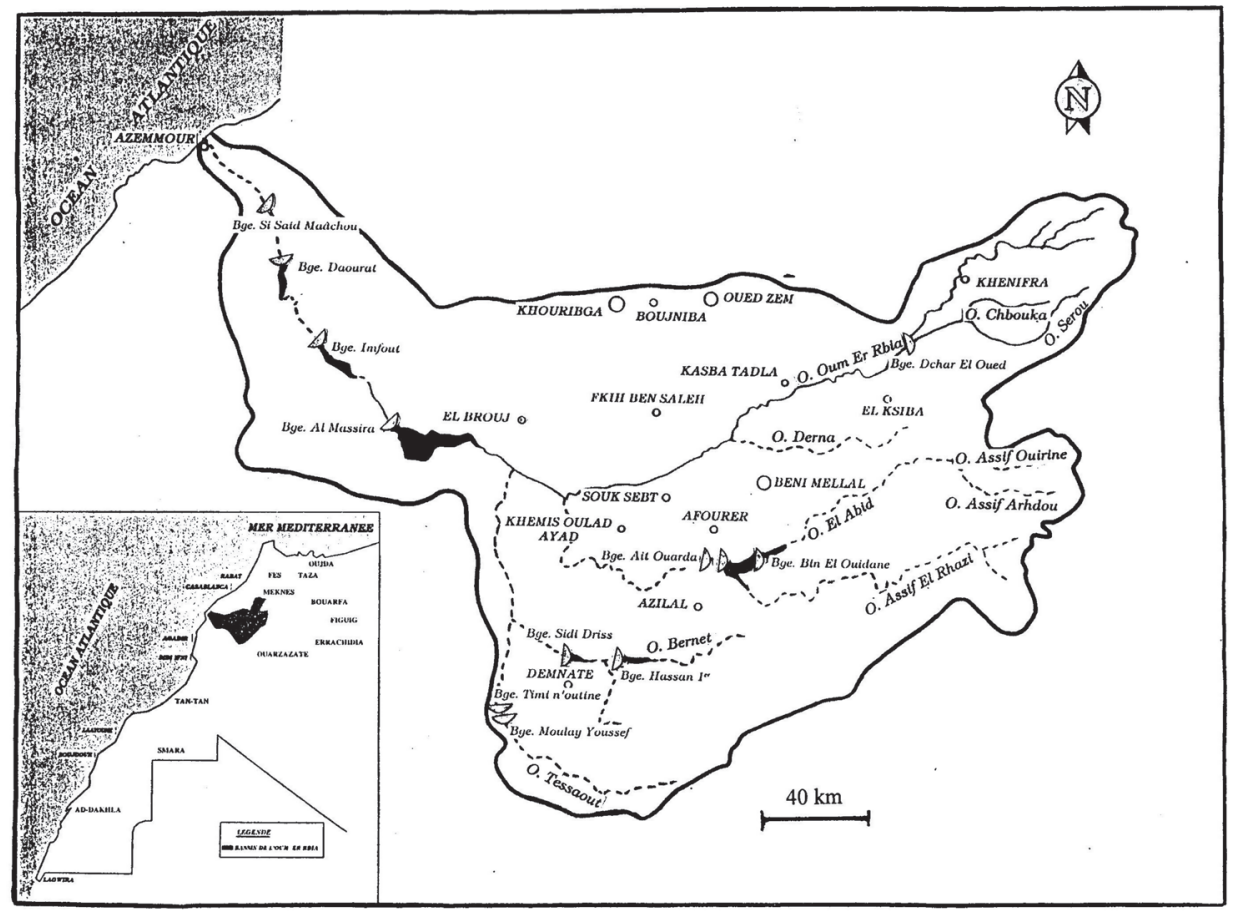

Fig. 1. - Bassin versant de l'oued Oum Er Rbia.

Fig. 1. - Oum Er Rbia river. 
Les principales caractéristiques de cette retenue sont données par le tableau I (Moncef, 1993).

Les prélèvements d'eau et d'animaux planctoniques, la mesure de la production primaire ainsi que les ex-

Tableau I. - Caractéristiques morphométriques de la retenue Al Massira.

Table I. - Morphometric characteristics of the Al Massira reservoir.

\begin{tabular}{|l|l|}
\hline Paramètres & Valeurs \\
\hline Surface du bassin versant & $28.500 \mathrm{Km}^{2}$ \\
Profondeur maximale & $40 \mathrm{~m}$ \\
Profondeur moyenne & $20 \mathrm{~m}$ \\
Longueur & $30 \mathrm{Km}$ \\
Largeur & $10 \mathrm{Km}$ \\
Volume & $280106 \mathrm{~m}^{3}$ \\
\hline
\end{tabular}

périences de broutage ont été effectués à $-5 \mathrm{~m}$ au niveau de la station la plus profonde (35 à $40 \mathrm{~m}$ ) (Fig. 2) jugée représentative de la zone pélagique (Moncef et al., 1994).

\subsection{Etude des facteurs écologiques}

\subsubsection{Paramètres physiques}

- Température : la mesure de la température de l'eau, parallèlement à celle de l'air, a été faite à l'aide d'un thermomètre à mercure gradué au $1 / 10^{\text {ème }}$.

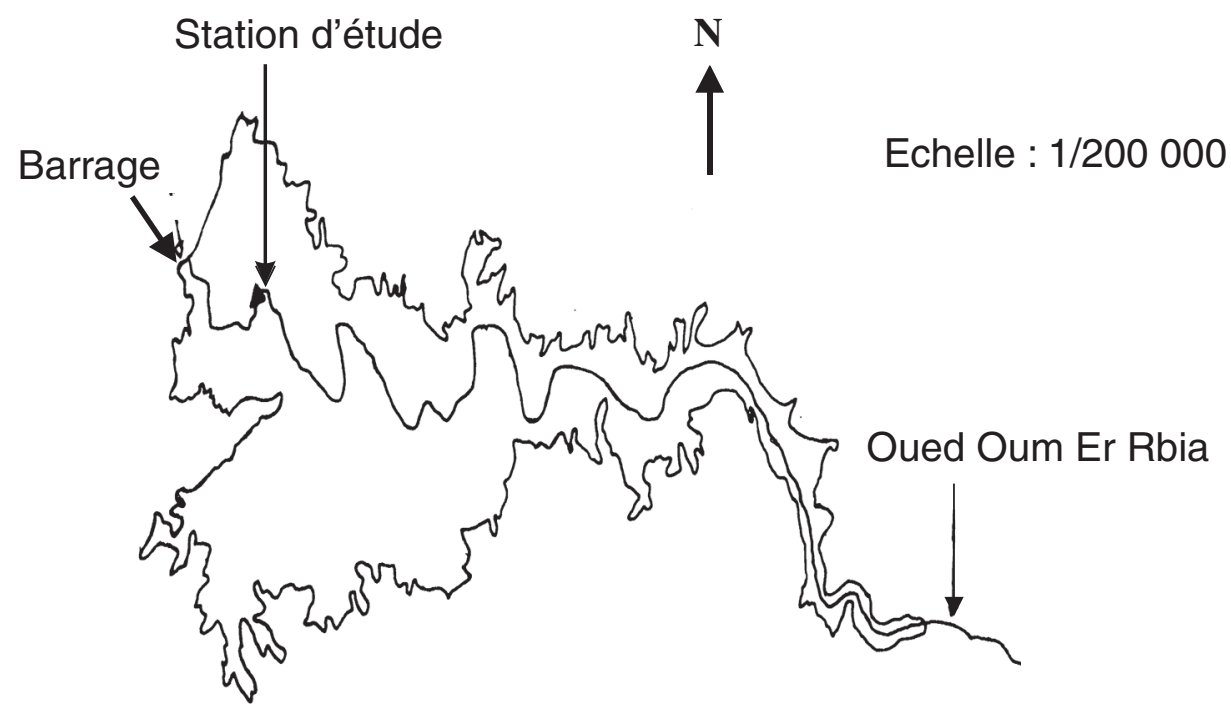

Fig. 2. - Morphologie et station d'étude de la retenue Al Massira (station située sur la ligne la plus profonde du réservoir).

Fig. 2. - Al Massira reservoir morphology and sampling station. 
- Conductivité : mesurée par un conductimètre de type Tacussel électronique.

- Turbidité : estimée à l'aide d'un disque de Secchi, les mesures ayant toujours été effectuées aux alentours du midi solaire (Clarke, 1941).

\subsubsection{Paramètres chimiques}

- $\mathrm{pH}$ : mesuré à l'aide d'un $\mathrm{pH}$-mètre de type WTW, permettant une lecture au $1 / 10^{\text {ème }}$ d'unité.

- Oxygène dissous: sa teneur, exprimée en $\mathrm{mg} / \mathrm{l}$, a été évaluée par la méthode volumétrique de Winkler.

- Les chlorures : Les teneurs en ions $\mathrm{Cl}^{-}$ont été dosés selon la méthode de MOHR, par le nitrate d'argent en présence du chromate de potassium utilisé comme indicateur coloré.

\subsubsection{Paramètres biologiques}

- Pigments chlorophylliens: les échantillons prélevés sont filtrés sur des filtres GF/C en microfibre de verre de l'ordre de $0,45 \mu \mathrm{m}$ de porosité. L'extraction des pigments chlorophylliens est réalisée par immersion des filtres dans de l'acétone $90 \%$ à l'obscurité et au frais pendant 24 heures. Les densités optiques de l'extrait acétonique sont mesurées à $760 \mathrm{~nm}$, puis à $663,645,630$ et $480 \mathrm{~nm}$ avant et après acidification à l'HCl $10 \%$. Les teneurs en chlorophylle, exprimées en $\mathrm{mg} \cdot \mathrm{m}^{-3}$, sont déter- minées à partir de l'équation de Lorenzen, (1967).

- Production primaire : La méthode utilisée est celle de l'oxygène dissous (méthode de Winkler) modifiée par Astelberg (Rodier, 1984). Elle consiste à immerger des flacons clairs et sombres à bouchons rodés de $250 \mathrm{ml}$, à diverses profondeurs et à mesurer les variations de la teneur en oxygène dissous après une durée d'incubation de six heures, autour du midi solaire.

\subsection{Expérimentation}

Le principe de la technique mise en œuvre lors de ce travail, consiste en une mesure de l'évolution des densités phytoplanctoniques au sein d'enceintes immergées in situ en présence (enceintes expérimentales) et en absence (enceintes témoins) de zooplancton.

Les enceintes utilisées, construites en plexiglas, sont de forme cubique et d'une capacité de 4,913 I. Sur cinq de leurs faces, sont placées dans un orifice de $14 \mathrm{~cm}$ de diamètre, des membranes filtrantes nucléopore de $4 \mu \mathrm{m}$ de porosité, permettant un renouvellement de l'eau (Moncef et al., 1994). Sur la sixième face (face supérieure), sont placés les dispositifs de fixation de l'enceinte sur le câble de soutien ainsi que l'ouverture de remplissage et de vidange (Moncef, 1993 ; Moncef et al., 1994). Nos expériences ont été réalisées à la profondeur $5 \mathrm{~m}$, à quatre périodes 
différentes à savoir octobre 1996, janvier 1997, avril 1997 et juillet 1997, représentant les quatre saisons de l'année. Deux enceintes, une expérimentale et une témoin, sont alors remplies d'eau prélevée à la profondeur $5 \mathrm{~m}$. Les prélèvements ont été réalisés à l'aide d'une bouteille fermante d'une capacité de 2,5 litres. Avant l'incubation et après avoir éliminé le zooplancton par filtration à l'aide d'une toile de $80 \mu \mathrm{m}$ dans l'enceinte témoin, $200 \mathrm{ml}$ d'eau sont prélevés de chacune des enceintes (témoin et expérimentale) et additionnés de lugol pour dénombrement des densités phytoplanctoniques initiales. Les enceintes sont ensuite immergées à la profondeur d'origine (5 m) et laissées en incubation pendant huit heures $(10 \mathrm{H}$ à $18 \mathrm{H})$.

A la fin de l'expérience, les mêmes quantités d'eau sont prélevées et additionnées de lugol pour la détermination des densités phytoplanctoniques finales sous microscope inversé. Le peuplement zooplanctonique présent dans l'enceinte expérimentale est récolté par filtration sur une toile en nylon de $80 \mu \mathrm{m}$ de porosité et immédiatement fixé au formol $4 \%$.

Les dénombrements ont été réalisés sur des cuves de Dolffus après coloration au Rose Bengal. Les effectifs spécifiques ont été convertis en biomasses à partir des relations longueur/poids établies par Dumont et al., (1975).

Le calcul du taux de prédation par unité de biomasse zooplanctonique a été réalisé à partir des hypothèses suivantes (Mayans, 1987) :
- dans les enceintes, la croissance phytoplanctonique est de type exponentiel ;

- à tout instant, la prédation s'effectue sur une fraction quantitativement constante du peuplement algal ;

- le taux de prédation est constant durant toute la période d'incubation.

Bien que l'eau soit soigneusement homogénéisée avant le remplissage des enceintes, les densités phytoplanctoniques sont susceptibles d'être différentes au temps zéro entre les enceintes témoins et expérimentales. Nous avons donc procédé aux dénombrements cellulaires dans chacune des enceintes afin de prendre en compte cette éventuelle hétérogénéité qui s'ajoute aux inévitables incertitudes liées aux dénombrements.

Dans ces conditions, pour chaque espèce phytoplanctonique, le taux de croissance spécifique intrinsèque $(u)$ peut être calculé à partir des informations obtenues dans l'enceinte témoin selon la relation :

$$
\mathrm{N}_{\mathrm{Tt}}=\mathrm{N}_{\mathrm{TO}} \mathrm{e}^{\mathrm{ut}}
$$

D'où : $u=\left(\log _{\mathrm{Tt}}-\log N_{\mathrm{TO}}\right) / \mathrm{t}$

Avec:

$\mathrm{N}_{\mathrm{Tt}}$ : nombre de cellules au temps $\mathrm{t}$ dans l'enceinte témoin ;

$\mathrm{N}_{\text {то }}$ : nombre de cellules au temps $\mathrm{t}_{0}$ dans la même enceinte.

Dans l'enceinte expérimentale, le taux de croissance $r$, résultante de la croissance intrinsèque $(u)$ et de la prédation $(p)$, est donné par :

$$
\mathrm{N}_{\mathrm{Et}}=\mathrm{N}_{\mathrm{E} 0} \mathrm{e}^{\mathrm{rt}}
$$


D'où : $r=\left(\log N_{E t}-\log N_{E 0}\right) / t$, avec : $\mathrm{N}_{\mathrm{Et}}=$ nombre de cellules au temps $\mathrm{t}$ dans l'enceinte expérimentale ;

$\mathrm{N}_{\mathrm{E} 0}=$ nombre de cellules dans la même enceinte en début d'incubation.

Or, $p=u-r$; la quantité totale des cellules ingérées par chaque espèce, est donc l'intégrale de la courbe représentant le nombre de cellules ingérées à chaque instant. II est possible d'estimer ce nombre par la relation :

$$
P=\left[\left[p\left(N_{E 0}+N_{E t}\right)\right] / 2\right] \cdot 1 / B
$$

où $P$ est la quantité ingérée par unité de biomasse zooplanctonique (B) et par unité de temps. C'est une approximation du taux de prédation moyen.

\section{RÉSULTATS ET DISCUSSION}

\subsection{Facteurs abiotiques}

Durant les quatre périodes d'étude, nous avons constaté que les eaux de la retenue, essentiellement alimentées par l'oued Oum Er Rbia, présentent des caractéristiques abiotiques plus ou moins variables selon le paramètre considéré.

Globalement, elles se caractérisent par l'absence de stratification thermique (sauf en juillet 97) (Fig. 3a), où l'écart thermique entre les eaux superficielles et celles du fond atteint $7^{\circ} \mathrm{C}$.

Les valeurs du $\mathrm{pH}$ sont maximales dans les dix premiers mètres, ce qui traduit l'influence de l'activité photosynthétique (Fig. 3b).

Les teneurs en oxygène dissous varient considérablement aussi bien dans le temps que dans l'espace (Fig. 3c). Les fortes valeurs observées dans la zone trophogène $\left(12,16 \mathrm{mg}^{-l^{-1}}\right.$ en janvier 97 à $5 \mathrm{~m}$; $10,72{\mathrm{mg} . \mathrm{I}^{-1}}^{\mathrm{en}}$ juillet 97 à la même profondeur) indiquent la prédominance de la production primaire sur les activités cataboliques. Les faibles teneurs observées dans les couches profondes (4 mg. $\mathrm{I}^{-1}$ en avril 97 et $2,56 \mathrm{mg} . \mathrm{I}^{-1}$ en juillet à $35 \mathrm{~m}$ ), témoignent d'une activité bactérienne intense (Malki, 1994).

En toute saison, les eaux sont faiblement minéralisées. La conductivité varie peu dans le temps et dans l'espace (Fig. 3d), la valeur maximale étant enregistrée en octobre 96 $\left(240 \mu \mathrm{s} . \mathrm{cm}^{-1}\right.$ dans toute la colonne d'eau) et la valeur minimale en janvier $\left(100,8 \mu \mathrm{s} . \mathrm{cm}^{-1}\right.$ à $\left.25 \mathrm{~m}\right)$.

\subsection{Facteurs biotiques}

Des teneurs élevées en chlorophylle a, sont observées en octobre $96\left(34,5 \mathrm{mg} \cdot \mathrm{m}^{-3}\right)$ et en janvier 97 $\left(20,25 \mathrm{mg}^{-3}\right)$ à $2 \mathrm{~m}$ (Fig. $\left.4 \mathrm{a}\right)$, les teneurs minimales sont observées en juillet $97\left(0,25 \mathrm{mg} \cdot \mathrm{m}^{-3}\right)$ à 10 et à $35 \mathrm{~m}$.

La production primaire décroît en fonction de la profondeur (Fig.4b), les valeurs, variant entre $240 \mathrm{mg} \cdot \mathrm{m}^{-3} \cdot \mathrm{h}^{-1}$ en janvier 97 à $2 \mathrm{~m}$ et $18 \mathrm{mg} \cdot \mathrm{m}^{-3} \mathrm{~h}^{-1}$ en juillet à $25 \mathrm{~m}$, sont sous la dépendance d'un complexe paramétrique agissant en étroite interaction: la 

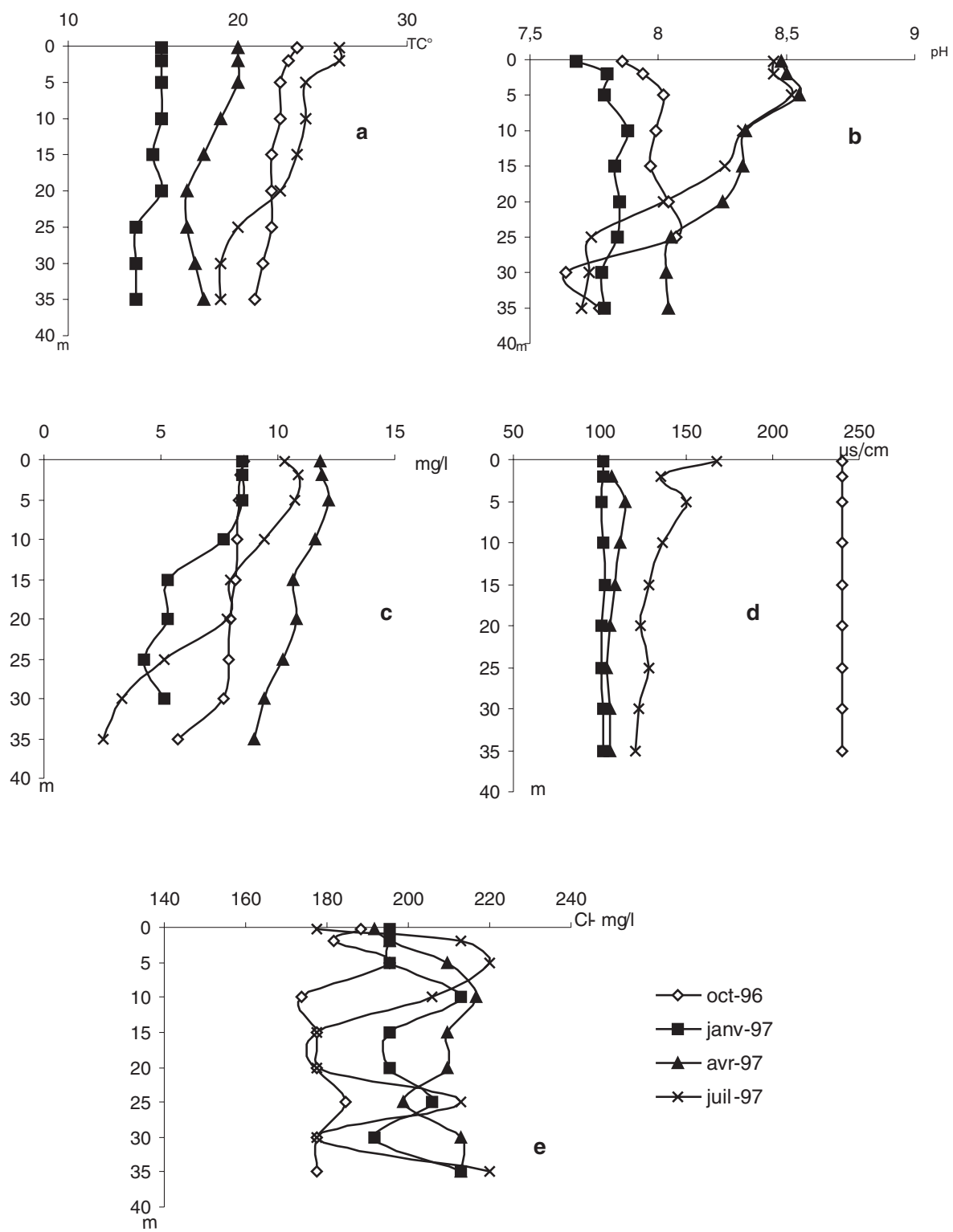

Fig. 3. - Profils verticaux de température (a), pH (b), oxygène dissous (c), conductivité (d) et chlorures (e) durant les périodes d'étude.

Fig. 3. - Spatio-temporal variations of temperature (a), $\mathrm{pH}(\mathrm{b})$, dissolved oxygen (c), conductivity (d) and chlorure (e) during the study periods. 

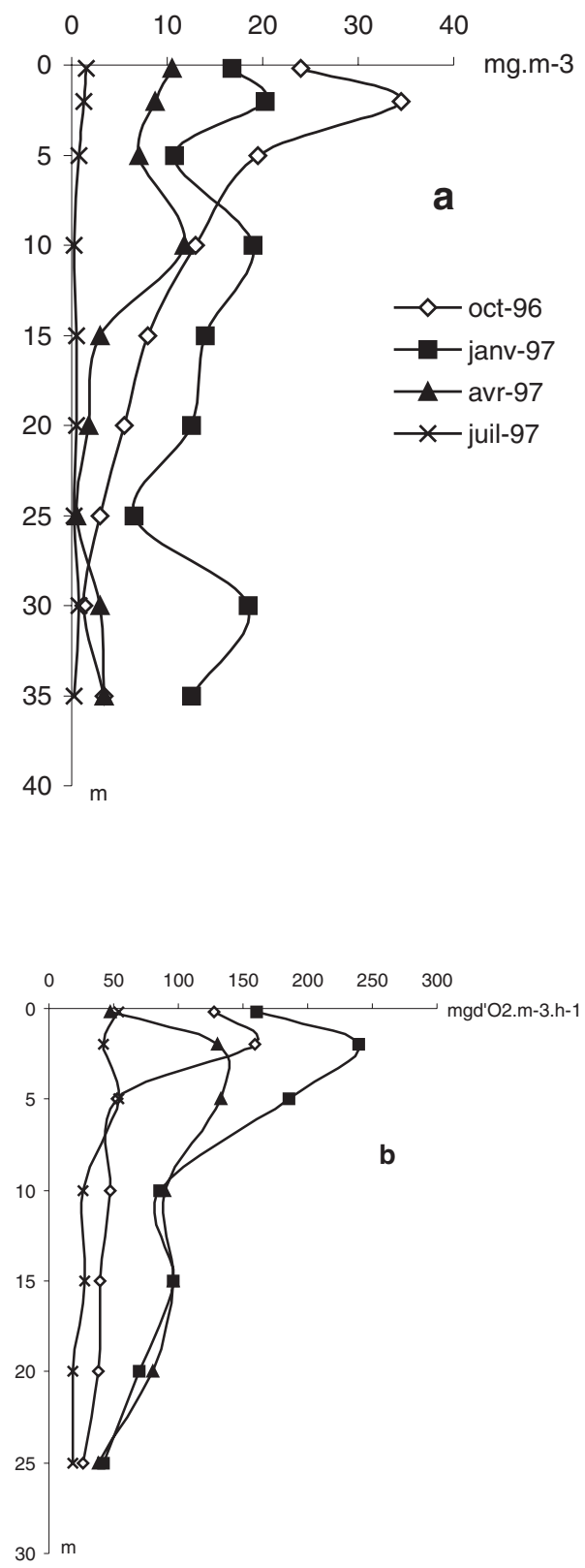

Fig. 4. - Profils verticaux de la teneur en chlorophylle (a) et de la production primaire (b). Fig. 4. - Spatio temporal variations of the chlorophyll a (a) and the primary production (b). pénétration de la lumière et la prédation par le zooplancton (Capblanc, 1974).

Le peuplement phytoplanctonique varie qualitativement et quantitativement selon les périodes d'étude (Fig. 5) :

- En octobre 96, les espèces dominantes sont: Oocystis crassa $\left(15,35.10^{4}\right.$ ind. $\left.l^{-1}\right)$, Cosmarium $s p$ $\left(18,84.10^{4} \mathrm{ind} / \mathrm{l}\right)$ et Chodatella $s p$ $\left(10,3.10^{4} \mathrm{ind} / \mathrm{l}\right)$.

- En janvier 97, Cryptomonas ovata qui domine $\left(10,52.10^{4}\right.$ ind. $\left.I^{-1}\right)$ suivi de Tetraedron minimum $\left(2,7.10^{4}\right.$ ind. $\left.I^{-1}\right)$.

- En avril 97, Cyclotella sp est l'espèce dominante $\left(75,66.10^{4}\right.$ ind..$\left.^{-1}\right)$.

- En juillet, nous avons noté la dominance d'Oocystis crassa $\left(28,55.10^{4}\right.$ ind. $\left.^{-1}\right)$ et de Melosira granulata $\left(13,65.10^{4}\right.$ ind.I $\left.{ }^{-1}\right)$.

Le peuplement zooplanctonique de la retenue est essentiellement représenté durant les périodes d'étude à la profondeur $5 \mathrm{~m}$, par trois espèces de copépodes: Neolovenulla alluaudi, Acanthocyclops robustus et Tropocyclops prasinus, quatre espèces de cladocères: Daphnia longispina, Ceriodaphnia reticulata, Diaphanosoma brachyrum et Bosmina longirostris, les rotifères ne constituant qu'une fraction minime (Fig. 6). L'abondance de chaque espèce varie en fonction des saisons (Fig. 7).

Lors des expérimentations réalisées à différentes périodes, les résultats (Tableau II et Tableau III) nous montrent que :

- En octobre 96, l'intensité du broutage est élevée; sur les vingt 


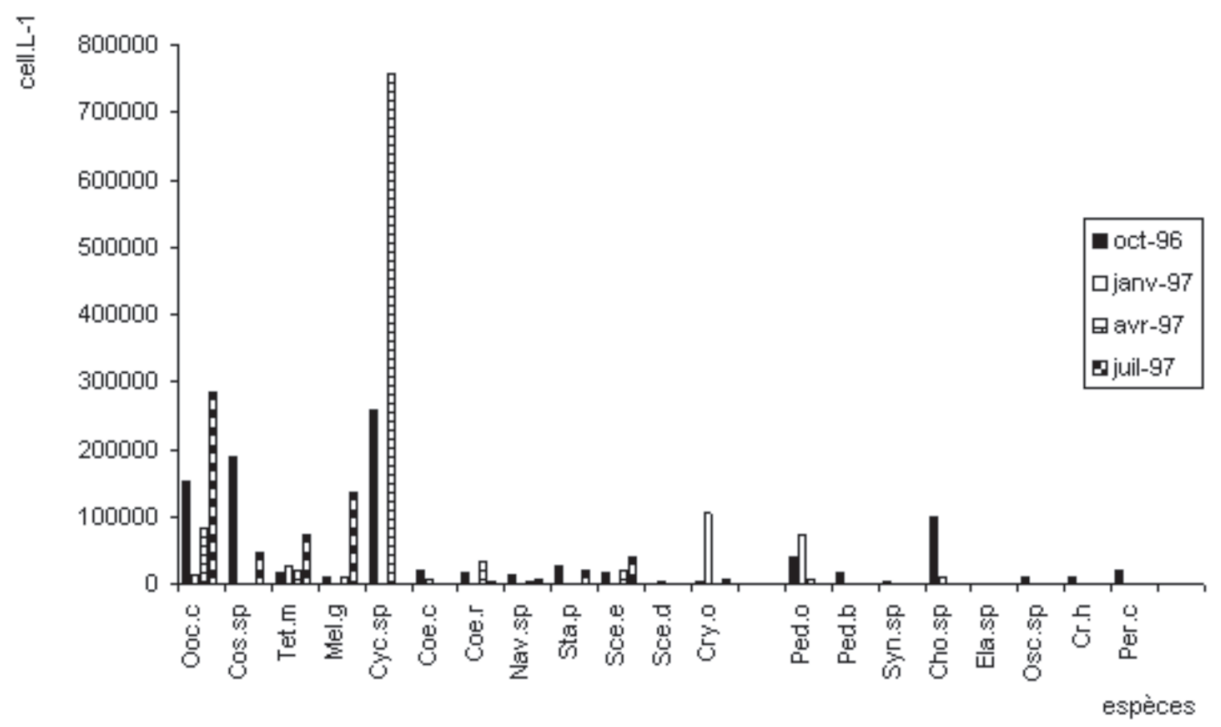

Fig. 5. - Densité des espèces phytoplanctoniques à $5 \mathrm{~m}$ durant les périodes d'étude.

Fig. 5. - Abundance of phytoplankton species at $5 \mathrm{~m}$ during the study periods.

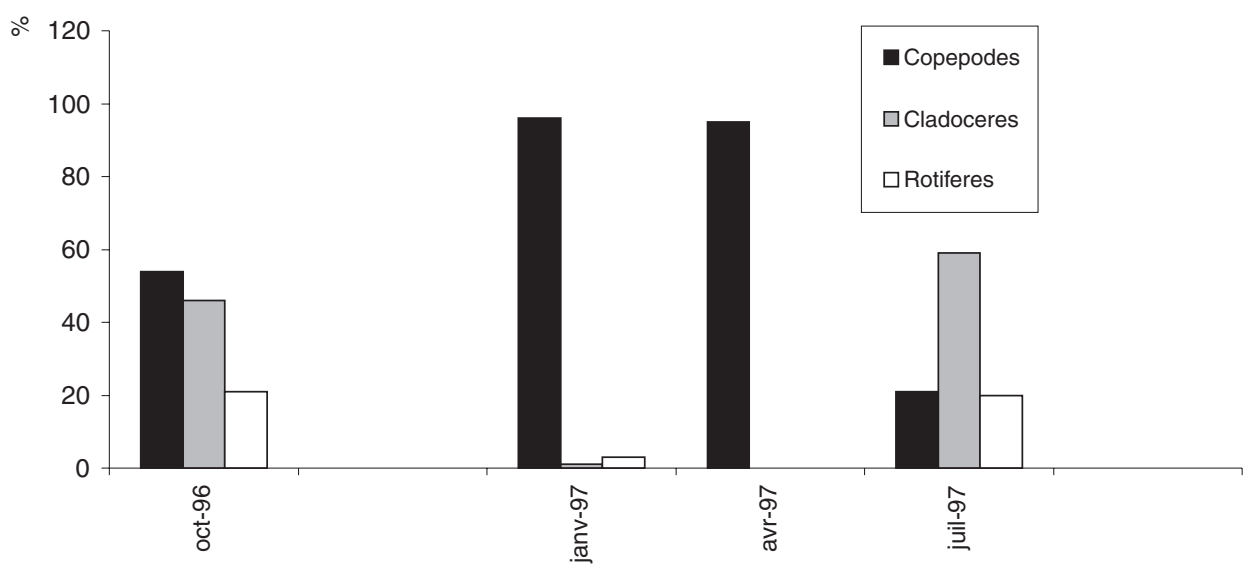

Fig. 6. - Biomasse (en \%) des copépodes, des cladocères et des rotifères durant la période d'étude.

Fig. 6. - Biomass (as \%) of copepods, cladocers and rotifers during the study periods. 


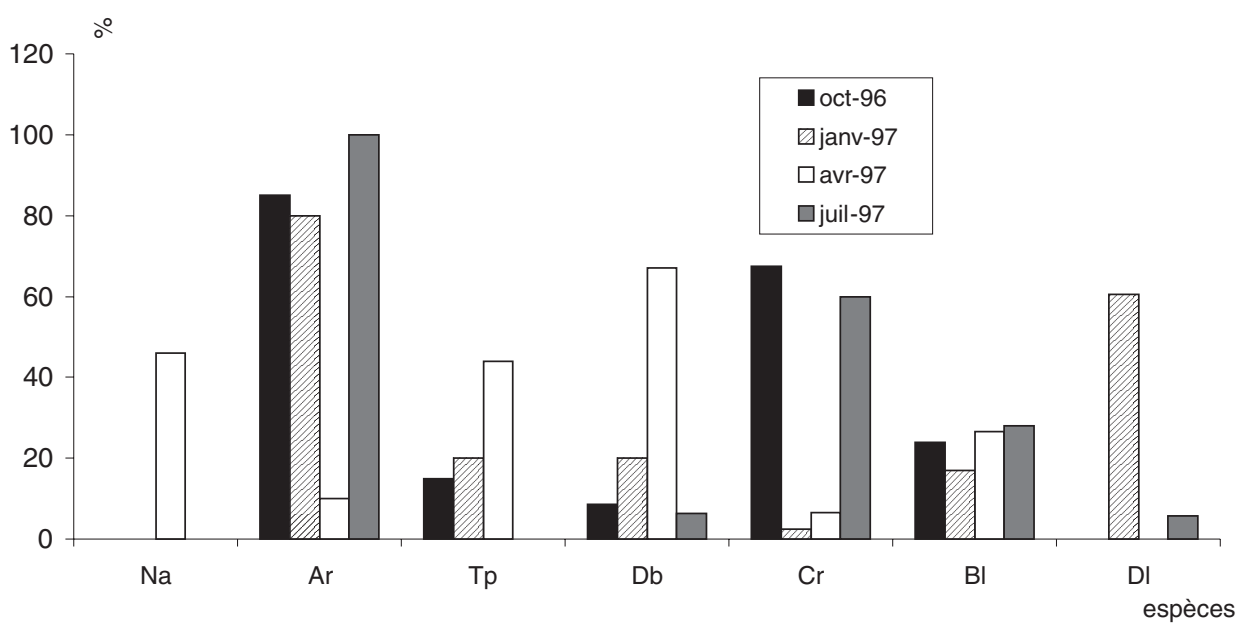

Fig. 7. - Abondance spécifique (en \%) des différentes espèces zooplanctoniques durant les périodes d'étude ( $\mathrm{Na}, \mathrm{Ar}, \mathrm{Tp}, \mathrm{Db}, \mathrm{Cr}, \mathrm{Bl}, \mathrm{DI}$ ).

Fig. 7. - Specific abondance (as \%) of different zooplanktonic species during the study periods ( $\mathrm{Na}$, $A r, T p, D b, C r, B I, D I)$.

Tableau II. - Évolution de la densité du peuplement phytoplanctonique (en \% de $\mathrm{N}_{\text {то }}$ ), en absence de zooplancton, après huit heures d'incubation.

Table II. - Changes in the abundance on phytoplankton cells $\left(\right.$ as $\%$ of $\mathrm{N}_{\mathrm{T}_{0}}$ ), when the flasks were free of the zooplankton over 8 hours of incubation.

\begin{tabular}{|c|c|c|c|c|}
\hline & $\% \mathrm{~N}_{\mathrm{T} 0}(\mathrm{NT})$ & $\% \mathrm{~N}_{\mathrm{T} 0}(\mathrm{NT})$ & $\% \mathrm{~N}_{\mathrm{T} 0}(\mathrm{NT})$ & $\% \mathrm{~N}_{\mathrm{T} 0}(\mathrm{NT})$ \\
\hline & oct-96 & jan-97 & avr-97 & juil-97 \\
\hline Oo.c & 157 & 118 & 108 & 142 \\
\hline Cos.sp & 122 & 186 & 0 & 132 \\
\hline Cyc.sp & 119 & 190 & 113 & 143 \\
\hline Tet.m & 125 & 0 & 113 & 112 \\
\hline Mel.g & 115 & 0 & 116 & 0 \\
\hline Coe.c & 103 & 0 & 0 & 0 \\
\hline Coe.r & 130 & 183 & 0 & 105 \\
\hline Sta.p & 110 & 0 & 0 & 113 \\
\hline Sc.e & 164 & 176 & 185 & 141 \\
\hline Cry.o & 230 & 116 & 0 & 133 \\
\hline Dict.p & 108 & 0 & 0 & 102 \\
\hline Ped.b & 89 & 98 & 0 & 97 \\
\hline Ped.o & 75 & 0 & 121 & 0 \\
\hline Cho.sp & 202 & 150 & 93 & 144 \\
\hline Ela.sp & 146 & 0 & 0 & 0 \\
\hline Cer.h & 0 & 0 & 0 & 0 \\
\hline Cer.c & 104 & 0 & 0 & 0 \\
\hline
\end{tabular}


Tableau III. - Evolution de la densité du peuplement phytoplanctonique (en \% de $\mathrm{N}_{\mathrm{To}_{\mathrm{O}}}$ ), en présence de zooplancton, après huit heures d'incubation.

Table III. - Changes in the abundance on phytoplankton cells $\left(\right.$ as $\%$ of $\mathrm{N}_{\mathrm{T}}$ ), when the flasks were contained of the zooplankton over 8 hours of incubation.

\begin{tabular}{|c|c|c|c|c|}
\hline & $\% \mathrm{~N}_{\mathrm{T} 0}(\mathrm{NE})$ & $\% \mathrm{~N}_{\mathrm{T} 0}(\mathrm{NE})$ & $\% \mathrm{~N}_{\mathrm{T} 0}(\mathrm{NE})$ & $\% \mathrm{~N}_{\mathrm{T} 0}(\mathrm{NE})$ \\
\hline & oct-96 & jan-97 & avr-97 & juil-97 \\
\hline Ooc.c & 64 & 62 & 46 & 85 \\
\hline Cos.sp & 88 & 60 & 82 & 61 \\
\hline Cyc.sp & 50 & 48 & 94 & 60 \\
\hline Tet.m & 57 & 0 & 43 & 0 \\
\hline Mela.g & 94 & 0 & 120 & 0 \\
\hline Coe.c & 72 & 0 & 0 & 116 \\
\hline Coer & 62 & 47 & 60 & 109 \\
\hline Sta.p & 112 & 0 & 0 & 50 \\
\hline Sce.e & 80 & 38 & 41 & 37 \\
\hline Cryp.o & 84 & 79 & 0 & 88 \\
\hline Dic.p & 45 & 0 & 0 & 93 \\
\hline Ped.b & 95 & 90 & 0 & 0 \\
\hline Ped.o & 120 & 0 & 0 & 0 \\
\hline Cho.sp & 67 & 64 & 75 & 0 \\
\hline Ela.sp & 86 & 0 & 0 & 0 \\
\hline Cer.h & 0 & 0 & 0 & 0 \\
\hline Cer.c & 108 & 0 & 0 & 0 \\
\hline
\end{tabular}

espèces phytoplanctoniques dénombrées, onze présentent des variations différentielles de densité avec le témoin entre le début et la fin de l'expérience. II s'agit de : Oocystis crassa (Ooc. c) ; Cosmarium sp (Cos. Sp) ; Cyclotella sp (Cyc. s); Tetraedron minimum (Tet. m) ; Colastrum cambricum (Cœ. c) ; Cœlastrum reticulatum (Cœ.r); Scenedesmus ecornis (Sce. e) ; Cryptomonas ovata (Cry. o) ; Dictyosphaerum pulchellum (Dyc. p); Chodatella $s p($ Cho, sp) ; Elakathotrix sp. (Ela. sp).
La plus forte pression de prédation (Fig. 8) s'exerce sur la diatomée Cyc. sp $\left(224,2\right.$ cel. $\left.\mu \mathrm{g}^{-1} \mathrm{~h}^{-1}\right)$ et la chlorophycée Ooc. $c\left(193,3\right.$ cel. $\left.\mu \mathrm{g}^{-1} \mathrm{~h}^{-1}\right)$.

L'importance du broutage au cours de cette période peut être liée à la coexistence de deux groupes majeurs : les copépodes représentant $54 \%$ de la biomasse zooplanctonique totale, et les cladocères représentant $45 \%$ (Fig. 5 et 6). Les copépodes sont dominés par Acanthocyclops robustus (85\% de la biomasse totale des copépodes). Chez les cladocères, c'est 


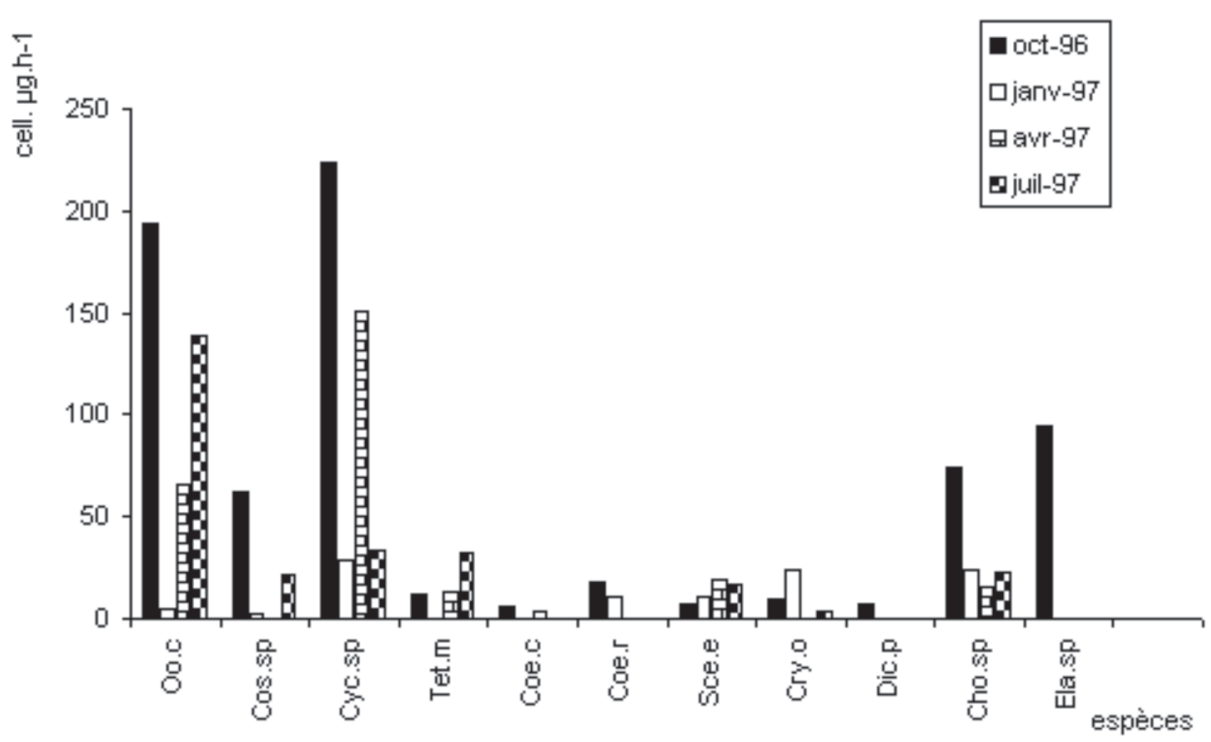

Fig. 8. - Intensité du broutage du peuplement phytoplanctonique par le zooplancton durant les périodes d'étude.

Fig. 8. - Intensity of the grazing pressure exerted by zooplankton on algae during the study period.

Ceriodophnia reticulata qui domine $(67,5 \%$ de la biomasse totale des cladocères). Il est ainsi très probable que la plus grande partie du broutage soit en liaison avec ces deux espèces.

- En janvier 97, nous remarquons que la pression de prédation s'est exercée sur six espèces phytoplanctoniques: Ooc. c., Cyc. sp, Cœ.r., Sce. e., Cry.o. et Cho. sp. L'intensité du broutage est très faible par rapport à la période précédente, la plus forte pression de prédation est observée chez Cyc.sp (28,6 cel. $\left.\mu \mathrm{g}^{-1} \cdot \mathrm{h}^{-1}\right)$, suivi de Cry. o. et Cho. sp $\left(24,4\right.$ cel. $\left.\mu g^{-1} \cdot h^{-1}\right)$. Cette faible intensité du broutage est en rapport avec la composition de la communauté zooplanctonique. Les copépodes représentent, à cette période, $96 \%$ de la biomasse zooplanctonique totale, l'espèce dominante étant Acanthocyclops robustus (80\% de la biomasse totale des copépodes).

- En avril 97, l'intensité du broutage est plus élevée par comparaison avec la période précédente (janvier 97). Cinq espèces phytoplanctoniques présentent de nettes variations de 
densité entre le début et la fin de l'expérience et par conséquent, des taux de prédation importants: Cyc $s p$ $\left(150,5 \mathrm{cel} . \mu \mathrm{g}^{-1} \cdot \mathrm{h}^{-1}\right), \quad$ Ooc. $\quad c \quad(65,5$ cel. $\left.\mu \mathrm{g}^{-1} \cdot \mathrm{h}^{-1}\right)$, Chod. sp $(15,84$ cel. $\left.\mu \mathrm{g}^{-1} \cdot \mathrm{h}^{-1}\right)$, Tet. $m\left(13,5 \mathrm{cel} . \mu \mathrm{g}^{-1} \cdot \mathrm{h}^{-1}\right)$ et Sce. e $\left(10,3\right.$ cel. $\left.\mu g^{-1} \cdot h^{-1}\right)$. Au cours de cette période, les copépodes constituent $97 \%$ de la biomasse zooplanctonique totale avec une dominance de Neolovenulla alluaudi (46\% de la biomasse totale des copépodes), suivi par Tropocyclops prasinus (44\%). Les cladocères sont dominés par Daphnia longispina (67\% de la biomasse totale des cladocères).

- En juillet 97, la plus forte pression de prédation a été enregistrée chez Ooc. $c\left(139,2\right.$ cel. $\left.\mu \mathrm{g}^{-1} \cdot \mathrm{h}^{-1}\right)$ et Cyc sp. $\left(33,5\right.$ cel. $\left.\mu \mathrm{g}^{-1} \cdot \mathrm{h}^{-1}\right)$. Durant cette période, les cladocères représentent $59 \%$ de la biomasse zooplanctonique, Ceriodaphnia reticulata étant l'espèce dominante $(60 \%$ de la biomasse totale des cladocères), suivi de Bosmina longirostris (28\%). Les copépodes représentent quant à eux $20 \%$, avec une seule espèce dominante : Acanthocyclops robustus.

L'intensité du broutage dépend des conditions biotiques et abiotiques de la retenue Al Massira, ce qui a été observée au sein de cette même retenue, par Moncef, (1993) ; Moncef et al., (1994), Fahde (1994) et Malki (1994). Des constatations similaires ont été faites dans d'autres milieux (Lair \& Oulad Ali, 1990; Sager \& Richman, 1991 ; Tifnouti et al., 1994 ; Francisco \& Rey, 1994).
Nos résultats confirment que le broutage est réellement sélectif, plusieurs espèces algales n'étant pas consommées (Nav. sp; Sta. $p$; Dic. $p$; Syn. $s p$; Cer. $h$; Per. c...). Ceci a été démontré auparavant par plusieurs auteurs (Gawler \& Angeli, 1987 ; Borsheim \& Anderson, 1987 ; Waleed et al., 1993 ; Moncef et al., 1994).

La dimension des cellules semblent être un paramètre influençant nettement la sélectivité de broutage. En effet, seules les algues de petite taille représentent des variations importantes d'effectifs dans les enceintes expérimentales entre les temps final et initial de chacune des quatre expérimentations. Conformément aux données de la littérature, la taille des cellules phytoplanctoniques apparaît bien, en raison des capacités mécaniques de capture des proies, comme l'un des critères majeurs de sélection par les crustacés planctoniques, en particulier chez les copépodes. En effet, ces derniers paraissent capables de sélectionner leurs proies entre les particules présentes dans leur environnement en fonction de la qualité de la nourriture, grâce à des chimiorécepteurs situés sur les appendices céphaliques et le labre, en adaptant un mode de collecte, filtration ou capture active, à la concentration et à la taille des particules (Moncef et al., 1994). La concentration des particules semble aussi un paramètre important de cette sélectivité, ceci a été observé chez certains copépodes (Gaudy, 1974 ; Tomasine \& Mazze, 1979). 


\section{CONCLUSION}

Au terme de ce travail, nous pouvons conclure que :

- L'intensité du broutage zooplanctonique sur le peuplement phytoplanctonique de la retenue $\mathrm{Al}$ Massira présente des variations saisonnières. Ces dernières dépendent des fluctuations, d'une part, de certains facteurs abiotiques tels que la température, le $\mathrm{pH}$, l'oxygène dissous, et d'autre part, de paramètres biologiques notamment la composition spécifique et l'abondance des populations phytoplanctoniques.

- Le broutage présente un impact très marqué sur le phytoplancton, particulièrement au printemps et en été.

- Le zooplancton exerce, par un broutage sélectif, une pression différentielle sur certaines espèces algales présentent dans le milieu.

\section{PERSPECTIVE}

A la lumière des résultats obtenus, nous envisageons dans un avenir proche de :

- Etudier séparément le broutage des copépodes et celui des cladocères ;

- Réaliser des expérimentations similaires sur d'autres écosystèmes lacustres marocains afin de procéder à des analyses comparées.

\section{REFERENCES BIBLIOGRAPHIQUES}

Borsheim (K.Y.) \& Anderson (R.S.), 1987 - Grazing and food selection by crustacean zooplankton in a shallow Norwegian Lake. J. Plankton Res., (9) : 367379.

Capblanc (J.), 1974 - Recherches sur la production primaire des lacs d'altitude. Doctorat es sciences. Uni. Paul sabatier. Toulouse.

Clarke (G.L.), 1941 - Obsevartions on transparency in the South - Wastern section of the North Atlantic ocean. J. mar. Res., 4(3) : 221-230.

Coveney (M.F.), Cronberg (G.) \& Enell (M.), 1977 - Phytoplankton, zooplankton and bacteria-standing crop and production relation ships in a eutrophic lake. Oiks., (29) : 5-21.

Dumont (H.J.), Van de Velde (J.) \& Dumont (I.), 1975 - The dry weight estimate of biomass in a selection of Cladocera, Copepoda and Rotifera from the plankton, periphyton and benthos of continental waters. Oecologia (Berl.), (19) : 75 - 97.

Fahde (A), 1994 - Etude des peuplements de Cladocères et rotifères dans le lac de barrage Al massira (Maroc). (Hétérogénéité horizontal, dynamique des populations, broutage). Thèse Doct. Etat, Univ. Hassan II. Casablanca.

Francisco (P.) \& Rey (J.), 1994 - Etude du peuplement zooplanctonique de retenue de Pareloup (Aveyron, France). Hydroecol. Appl.

Gaudy (R.), 1974 - Feeding four species of pelagic copepods under experimental conditions. Mar. Biol., (25) : 125141.

Gawler (M.) \& Angeli (N.), 1987 - Intensité du broutage dans le lac Léman en relation avec la structure de la taille 
des particules variation nycthémérales. Natural. Can. : 114.

Haney (J.F.), 1971 - An in situ method for measurement of zooplancton grazing rates. Limnol. Oceanogr., (16) : 970977.

Kiorboe (T.), 1985 - In situ feeding rates of planktonic copepods : A comparison of four methods. J. Exp. Mar. Biol., (88) : 67-81.

Lair (N.) \& Oulad Ali (H.), 1990 - Grazing and assimilation rates of natural populations of planktonic rotifers Keratella Cochlearis, Keratella quadrata and Kellicottia longispina in a eutrophic lake (Aydat, France), Hydrobiologia (194) : 119-131.

Lorenzen (N.C.J.), 1967 - Determination of chlorophyll and phaeopigments : spectrophotometric equation. Limnol. Oceanogr., 12(2) : 343-346.

Mackas (D.) \& Bohrer (R.), 1976 - Fluorescence analysis of zooplankton gut contents and investigation of diel feeding patterns. J. Exp. Mar. Biol. Ecol. : 77-85.

Malki (M.), 1994 - Etude de la communauté phytoplanctonique et des caractéristiques physico-chimiques des eaux du lac réservoir Al Massira. Thèse d'état es sciences, Univ. Hassan II. Casablanca.

Mayans (M.), 1987 - Etude expérimentale in situ du broutage par le zooplancton (Daphnia longispina) sur le peuplement algal printanier d'une retenue oligotrophe (Ste croix, Provence). DEA Université Aix-Marseille (1) : 61pp.

Moncef (M.), 1993 - Etude du peuplement de copépodes et des larves de chaoboridaes de la retenue de barrage Al Massira (Maroc). Thèse d'état es sciences Naturelles. Univ. Hassan II, Casablanca.

Moncef (M.), Malki (M.), Fahde (A.) \& Devaux (J.), 1994 - Mesure de l'intensité et la sélectivité du broutage zooplanctonique diurne et nocturne sur le peuplement phytoplanctonique de la retenue Al Massira (Maroc). Sciences de l'eau, (7) : 119-131.

Mourelatos (S.), 1989 - Broutage du phytoplancton par le zooplancton dans un lac peu profond. Thèse Doct. Univ. Pars 6 : 191pp.

Northcote (T.G.), Arcifa (M.S.) \& Munro (K.A.), 1990 - An experimental study of the effects of fish zooplanktivory on the phytoplankton of a Brazilian reservoir. Hydrobiologia., (194) : 31-45.

Oulad Ali (H.), 1988 - Rôle et importance des rotifères planctoniques dans un écosystème lacustre eutrophe de la zone tempérée Nord: le Lac d'Aydat (France) - Thèse de l'université Blaise Pascal de Clermont-Ferrand.

Paul (A.J.), Leavitt (P.R.), Schindler (D.W.) \& Hardie (A.K.), 1995 - Direct and indirect effects of predation by a calanoïd copepod (subgenus : Hesperodiaptomus) and of nutrients in a fishless alpine lake. Can. J. Fish. Aquat. Sci., (52) : 2628-2638.

Porter (K.G.), 1972 - A method for the in situ study of zooplankton grazing effects on species composition and standing crop. Limnol. Oceanogr., 17(6) : 913-977.

Rodier (J.), 1984 - L'analyse de l'eau. edit. Dunol : 1365pp

Sager (P.E.) et al., 1990 - Functional interaction of Phytoplancton and Zooplancton along the

Trophic Gradient in Green Bay ,Lake Michigan ${ }^{1}$. Can.J.Fish. Aquat. Sci.,(48) : 116-122.

Sager (P.E.) \& Richman (S.), 1991 Functional interaction of phytoplankton and zooplankton along the Trophic Gradient in Green Bay, lake Michigan. Can. J. Fish. Aquat. Sci., (48) : 116122.

Tifnouti (A.), Cherifi (O.) \& Chifa (A.), 1994 - Etude du régime alimentaire de cinq espèces de cladocères dans la retenue lalla Takerkoust (Maroc). Annls. Limnol. 30(4) : 285-296. 
Tomasine (J.A.) \& Mazze (J), 1979 Comportement alimentaire de Centropages typicus. (Copepoda, calanoïda) en milieux nutritifs à deux algues et en présence de Nauphii d'Artemia sp. J. Cons. Int. Explor. Mer., (38) : 293-307.

Vijverberg (J.), 1976 - The effect of food quantity and quality on the Growth, Birth-Rate and longevity of Daphnia
Hyalina Leydig. Hydrobiologia., 51(2) : 99-108.

Waleed (H), Manca (M) \& Ruggiu (D), 1993 - Field estimates of zooplankton community grazing in the Limed lago d'orta (Italy) by the use of a radioisotope-free technique. Hydrobiologia, (264) : 47-54.

\section{Liste des abréviations utilisées pour les espèces phytoplanctoniques}

\begin{tabular}{|c|c|c|c|}
\hline Ooc. c & : Oocyctis crassa & Cos. $s p$ & Cosmarium sp \\
\hline Cyc. sp & : Cyclotella sp & Tet. $m$ & : Tetraedron minimum \\
\hline Mel. $g$ & : Melosira granulata & Coe. $c$ & : Coelastrum cambricum \\
\hline Coe. $r$ & : Coelastrum reticulatum & Nav. sp & : Navicula $s p$ \\
\hline Sta. $p$ & : Staurastrum pingue & Sce. e & : Scenedesmus ecornis \\
\hline Sce. $d$ & : Scenedesmus denticulata & Cry. o & Cryptomonas ovata \\
\hline Dic. $p$ & : Dictyosphaerium pulchellum & Ped. $b$ & : Chodatella $s p$ \\
\hline Ela. sp & : Elakatothrix sp. & Cer. $h$ & : Ceratium hirundinella \\
\hline Osc. $s p$ & : Oscillatoria $s p$ & Ped. $b$ & : Pediastrum boryanum \\
\hline Ped. o & : Pediastrum ovatum & Syn. sp & : Synedrea sp \\
\hline Cho. sp & : Chodatella sp & Cer. $h$ & Ceratium hirundinella \\
\hline
\end{tabular}

\section{Liste des abréviations utilisées pour les espèces zooplanctoniques}

$\begin{array}{ll}\text { N.a } & \text { : Neolovenula alluaudi } \\ \text { A.r } & \text { : Acanthocyclops robustus } \\ \text { T.P } & \text { : Tropocyclops pracinus } \\ \text { D.b } & \text { : Diaphanosoma brachyrum } \\ \text { D.I } & \text { : Daphnia longispina } \\ \text { C.r } & \text { : Ceriodaphnia reticulata } \\ \text { B.I } & \text { : Bosmina logirostris }\end{array}$




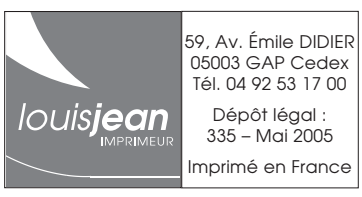

